\section{Simulation of $\mathrm{LHCb} \mathrm{RICH}$ Detectors using GEANT4}

\author{
I. Belyaev ${ }^{4)}$, G. Corti ${ }^{3)}$, S. Easo ${ }^{1)}$, \\ C. Jones ${ }^{2)}$, A. Papanestis ${ }^{1)}$, W. Pokorski ${ }^{3)}$, \\ F. Ranjard ${ }^{3)}$, P. Robbe ${ }^{5)}$ \\ For the LHCb Simulation Group.
}

\begin{abstract}
$\mathrm{LHCb}$ is one of the experiments at the Large Hadron Collider at CERN. It aims to make precision measurements of $\mathrm{CP}$ violation in the B-meson decays. The Detector simulation of $\mathrm{LHCb}$ was developed using the GEANT4 software toolkit interfaced to the $\mathrm{LHCb}$ software framework named GAUDI. This is now being used for a large production run of 210 million events. An important feature of the $\mathrm{LHCb}$ experiment is the use of Ring Imaging Cherenkov Counters (RICH) for particle identification. The Cherenkov photons created in this detector are focused onto an array of photodetectors made of Hybrid Photodiodes.

An brief overview of the GEANT4 based $\mathrm{LHCb}$ simulation program named GAUSS will be given with the main emphasis on RICH simulation and its verification.
\end{abstract}

\section{$1 \quad \mathrm{LHCb}$ and its $\mathrm{RICH}$ detectors}

The main goals of the $\mathrm{LHCb}$ experiment are to make precision measurements of the $\mathrm{CP}$ violation in B-Meson decays and to look for signs of the physics beyond the 'Standard Model' in particle physics. The detector is configured as a single forward arm spectrometer with open geometry, optimized for detecting B-hadrons produced in the Large Hadron Collider (LHC). It is expected to collect about $2 \times 10^{9} b \bar{b}$ events per year, from the startup of LHC.

LHCb consists of a silicon strip detector near the interaction region, a dipole magnet, a set

1) Rutherford Appleton Laboratory, Chilton, Didcot, Oxon OX11 OQX, UK.

2) University of Cambridge, Cavendish Laboratory, Madingley Road, Cambridge CB3 OHE, UK.

3) CERN, Department PH, 1211 Geneva 23, Switzerland.

4) Institute of Theoretical and Experimental Physics, Moscow, Russia.

5) LAL Orsay, IN2P3-CNRS and University of ParisSud, Orsay, France. of tracking detectors which use silicon sensors near the central region and drift chambers away from the central region, two RICH detectors, an electromagnetic calorimeter using lead plates interspersed with scintillator plates, a hadronic calorimeter using iron-scintillator tile technology and a muon detector system based on MWPCs and GEM chambers. A detailed description of $\mathrm{LHCb}$ can be found in [1], [2].

Particle identification is an essential component of the physics programme of LHCb. For example, separating pions and kaons using the RICH suppresses backgrounds coming from $B_{s} \rightarrow D_{s}^{ \pm} \pi^{\mp}$ when selecting the $B_{s} \rightarrow D_{s}^{ \pm} K^{\mp}$ decay mode. In this case, the background is 10 times more than the signal and the RICH reduces the background contamination to a $10 \%$ level. The RICH detector will also be used to tag the flavour of the B-hadrons by identifying the kaons in the events where the b-quark decays into an s-quark through the cascade decay $(b \rightarrow c \rightarrow s)$.

The RICH system in LHCb has two detectors. The first detector(RICH1) has aerogel and $C_{4} F_{10}$ as radiators, covering the full $\mathrm{LHCb}$ acceptance of $300 \mathrm{mrad}$ and is placed upstream of the $\mathrm{LHCb}$ dipole magnet in order to identify the particles with momenta between 2 and $70 \mathrm{GeV} / \mathrm{c}$. The second detector(RICH2) has $C F_{4}$ as radiator and is situated downstream of the magnet. It covers an acceptance of 120 $\operatorname{mrad}$ (horizontal) $\times 100 \mathrm{mrad}$ (vertical) and is for identifying particles with momenta up to $100 \mathrm{GeV} / \mathrm{c}$. Hybrid Photo Diodes(HPD) with $80 \mathrm{~mm}$ photocathode diameter will be used to detect the Cherenkov photons in the wavelength range $200-600 \mathrm{~nm}$. The HPDs encapsulate silicon pixel anodes bump bonded to readout chips.

\section{Detector Simulation of $\mathrm{LHCb}$}

Detector simulation is needed for preparing the analysis of the data to be collected and is an important part of developing the detectors in LHCb. Earlier versions of simulation written in FORTRAN using the GEANT3 package have been replaced with a more detailed simulation program using the GEANT4 [3] package. This new program named GAUSS has recently been used in a production run of 210 million events. The main features of GAUSS and the initial set of results from simulating the RICH detectors are the subjects of this paper. 


\subsection{Simulation Framework}

The GAUDI framework [4],[7] is used by $\mathrm{LHCb}$ for all its software applications, including simulation. Separation 'Data' from 'Algorithms' is one of the main features of GAUDI. Several GAUDI services facilitate different types of 'Data' available to be used in 'Algorithms' whenever needed.

GAUDI is interfaced to GEANT4 using the abstract interfaces set up in a package named GiGa. [8] . This package provides GAUDI with access to the various parts of GEANT4, including its main event loop. As a result, the GEANT4 is fully driven by GAUDI.

GAUSS is an application of GAUDI which uses GiGa and contains all the software for the simulation of the various detectors in $\mathrm{LHCb}$. The Geometry description of the $\mathrm{LHCb}$ is read from a database in XML format into a transient representation in GAUDI. From this, using the appropriate GAUDI service and the GiGa interface, the corresponding GEANT4 geometry description is created.

The minimum bias proton-proton interactions at $\sqrt{s} 14 \mathrm{TeV}$ are generated using the PYTHIA [5] package and the decay of most of the unstable hadrons are performed using the EVTGEN [6] package. The hadrons, leptons and photons generated from these are transferred into GEANT4 using the corresponding GAUDI service.

\subsection{Physics Processes}

The various electromagnetic and hadronic interactions of the particles traversing the various detectors are simulated by activating the appropriate set of GEANT4 physics processes. These processes are defined for each particle declared in GEANT4 and each process has its cross-section defined from a set of available models and experimental data. Particle decays are performed according to the branching ratios declared for each decay channel. GEANT4 has a large selection of physics processes and $\mathrm{LHCb}$ uses a selected list of processes similar to those used by other LHC experiments.

The HPDs in RICH are sensitive to the photons in the wavelength range $200-800 \mathrm{~nm}$. Hence the Cherenkov photons simulated in $\mathrm{RICH}$ are restricted to wavelengths near these limits and are classified as 'Opticalphotons'. They undergo Fresnel reflections and refractions at the various optical boundaries and activate photoelectric process at the photocathode of the HPDs. They are also subject to bulk absorption in various materials and Rayleigh scattering in aerogel. The photoelectrons created in the photocathode arrive at the Silicon detector of the Hybrid Photodiode. These electrons and other charged particles which traverse the Silicon detector create 'hits' and the information regarding these hits is stored in the output of the program.

The simulation of the photoelectric effect in the HPDs is a custom-made physics process which uses the quantum efficiency measurements of the HPDs to estimate the probability of photoelectron creation. The simulation of the energy loss process in the Silicon detector of HPD is also a custom-made physics process, optimized for speed.

\section{$3 \quad$ Validation of the Simulation}

To validate GAUSS, results from testbeam data analysis and the results from the earlier versions of the LHCb simulations are used whenever they are appropriate.

\subsection{Validation of RICH Simulation using testbeam}

A testbeam with $10 \mathrm{GeV} / \mathrm{c}$ pions at CERN PS was used to study the performance of aerogel as a Cherenkov radiator. The Cherenkov photons were detected by means of four large HPD tubes designed and constructed at CERN. A detailed description of this setup and the full set of results can be found in [9]. In two of the run configurations, data were taken with aerogel tiles of thickness $4 \mathrm{~cm}$ and $8 \mathrm{~cm}$. In these runs an optical filter was placed downstream of aerogel which cut off photons with wavelengths below $300 \mathrm{~nm}$ in order to reduce the 'chromatic' error in the Cherenkov angle resolution due to the large variation in the refractive index of aerogel at these wavelengths. In Table 1 , the measurements of the photoelectron yield and the reconstructed Cherenkov angle in these configurations are shown for the data simulated using GEANT4 and the real data. Good agreement is seen in this table between the results from real data and simulation.

\subsection{Comparison of RICH Simulation in GAUSS with earlier results.}

For the LHCb-RICH detector, the earlier versions of the simulation used GEANT3 for the tracking of charged particles and in-house software for simulating the creation and propagation of Cherenkov photons. The GAUSS is designed 


\begin{tabular}{|c|c|c|}
\hline $\begin{array}{c}\text { Aerogel } \\
\text { Thickness }\end{array}$ & $\begin{array}{c}\text { Yield } \\
\text { Per Event }\end{array}$ & $\begin{array}{c}\text { Cherenkov } \\
\text { Angle in mrad }\end{array}$ \\
\hline $4 \mathrm{~cm}$ Data & $6.3 \pm 0.7$ & $247.1 \pm 5.0$ \\
\hline $4 \mathrm{~cm}$ MC & $7.4 \pm 0.8$ & $246.8 \pm 3.1$ \\
\hline $8 \mathrm{~cm}$ Data & $9.4 \pm 1.0$ & $245.4 \pm 4.8$ \\
\hline $8 \mathrm{~cm}$ MC & $10.1 \pm 1.1$ & $243.7 \pm 3.0$ \\
\hline
\end{tabular}

Table 1: Results from RICH testbeam in terms of the Photoelectron yield per event and the reconstructed Cherenkov angle. These are compared with the corresponding results from GEANT4 simulation.

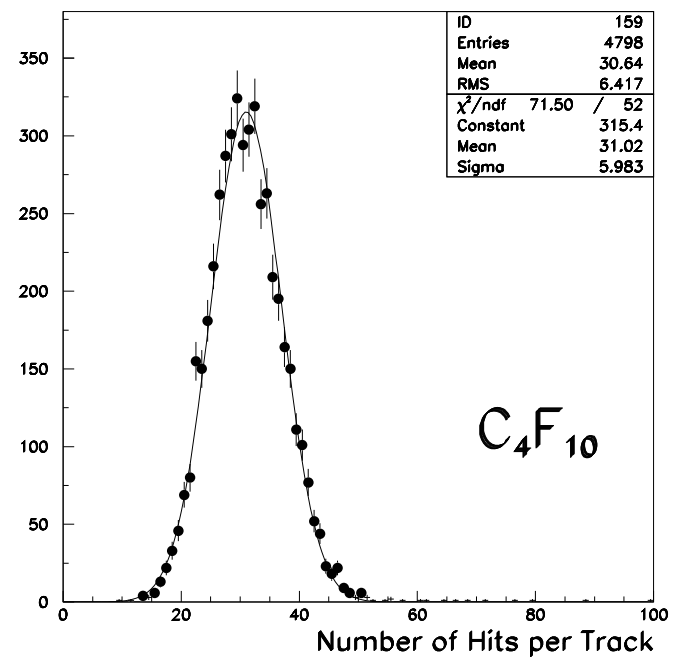

Figure 1: Photoelectron yield from GAUSS in $C_{4} F_{10}$ radiator for saturated tracks.

to have detector configurations which are more realistic and detailed than those in the earlier versions. As a result, the results from the two simulations are expected to be similar, but not identical.

The photoelectron yield obtained from GAUSS for saturated tracks (Beta $\simeq 1$ ) in the $C_{4} F_{10}$ radiator is plotted in Figure 1. In Table 2, the mean yield obtained from this distribution and the average yields obtained from the other two radiators are quoted along with the corresponding yields from the earlier simulations. The average yields obtained from the two simulations are similar for each of the three radiators, as shown in this table.

From the output of GAUSS, Cherenkov angles are reconstructed for each hit and compared with the corresponding expected value based on

\begin{tabular}{|c|c|c|}
\hline Radiator & $\begin{array}{c}\text { Old } \\
\text { Simulation }\end{array}$ & GAUSS \\
\hline Aerogel & 6.8 & 6.8 \\
\hline$C_{4} F_{10}$ & 30.3 & 31.0 \\
\hline$C F_{4}$ & 23.2 & 23 \\
\hline
\end{tabular}

Table 2: Mean Photoelectron yield from saturated tracks using GAUSS and the old simulation for the three radiators.

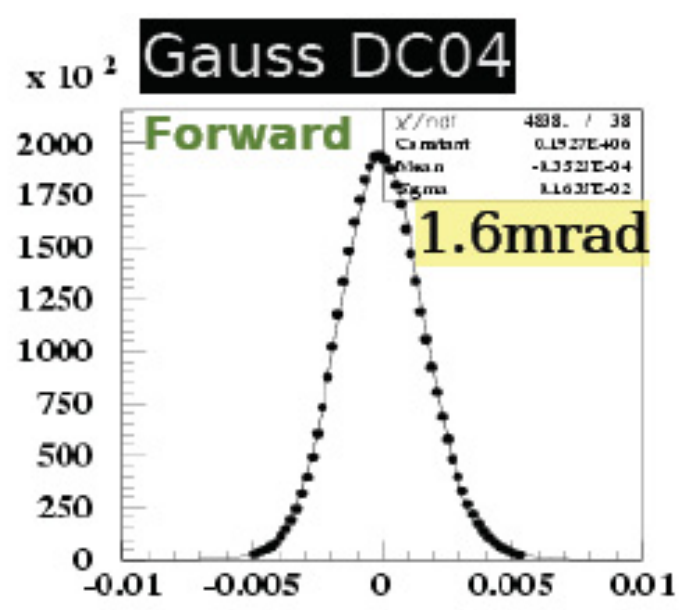

Figure 2: Difference in the Reconstructed and Expected estimates of the Cherenkov angles in $\mathrm{mrad}$ in the $C_{4} F_{10}$ radiator from data made by GAUSS. The distribution has a width of 1.6 mrad and is made only for the best measured tracks named 'FORWARD' tracks in LHCb.

track parameters. For this, in Figure 2 the difference between these two quantities is plotted for the $C_{4} F_{10}$ radiator for the best measured charged tracks. The widths of such distributions made for all three radiators are compared with those from earlier simulations in Table 3. Although the results from the two simulations are similar, the GAUSS shows a small degradation in performance. This is expected since both the RICH system and charged tracking system used more realistic simulations in GAUSS than those used in the old simulation.

From the output of GAUSS, particle identification is performed using a log likelihood method [10] and the efficiency of identifying pion as a light particle (pion, muon or electron) and the probability of misidentifying Kaon as a light particle are plotted in Figure $3 \mathrm{a}$ as a function of particle momentum from the GAUSS for the best measured charged tracks in minimum 


\begin{tabular}{|c|c|c|}
\hline Radiator & $\begin{array}{c}\text { Old } \\
\text { Simulation }\end{array}$ & GAUSS \\
\hline Aerogel & 2.6 & 2.6 \\
\hline$C_{4} F_{10}$ & 1.3 & 1.6 \\
\hline$C F_{4}$ & 0.58 & 0.61 \\
\hline
\end{tabular}

Table 3: Single photon Cherenkov Angle resolutions in mrad from GAUSS and old simulation for the three radiators. These were obtained for the best measured tracks types in LHCb.

bias events. In Figure $3 \mathrm{~b}$ the same plots obtained from the old simulation are shown. The plots from the two simulations are similar. The pion identification efficiency obtained using all charged track types in $B_{s} \rightarrow D_{s}^{ \pm} K^{\mp}$ events for different momentum ranges is shown in Table 4 from the GAUSS and the old simulation. This table also shows similar results from the two simulations. The small degradation in the efficiency from GAUSS can be explained by the fact that the GAUSS is set to be more realistic than the old simulation.

\begin{tabular}{|c|c|c|}
\hline $\begin{array}{c}\text { Momentum } \\
\text { Range } \\
\text { in GeV/c }\end{array}$ & $\begin{array}{c}\text { Old } \\
\text { Simulaton } \\
\text { Efficiency \% }\end{array}$ & GAUSS \\
Efficiency \% \\
\hline $2-10$ & $90.12 \pm 0.08$ & $89.84 \pm 0.06$ \\
\hline $10-70$ & $94.89 \pm 0.07$ & $94.76 \pm 0.04$ \\
\hline $70-100$ & $76.96 \pm 1.0$ & $76.94 \pm 0.70$ \\
\hline
\end{tabular}

Table 4: The efficiency (in \% ) of identifying pion as a light particle for all the track types in different momentum ranges, using the old Simulation and GAUSS.

\section{Summary}

LHCb simulation using GEANT4 is fully operational. A large production run named DC04, using this program has been completed recently. The RICH simulation using GEANT4 is more detailed and more realistic than that used in earlier versions of simulation. The results from GAUSS for the RICH are validated using results from earlier simulations and testbeam data. Physics analysis using the data simulated in DC04 is in progress.

\section{References}

[1] LHCb Reoptimized Detector Design and Performance CERN/LHCC 2003-030.

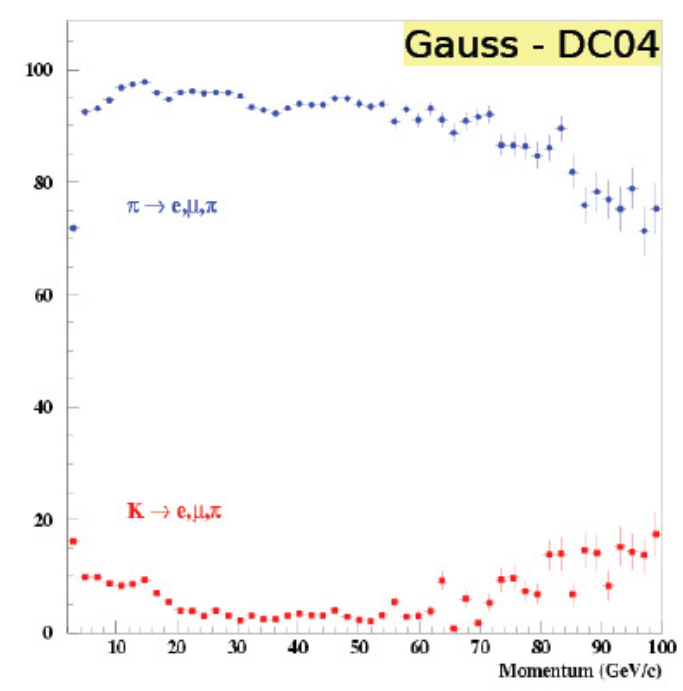

(3a)

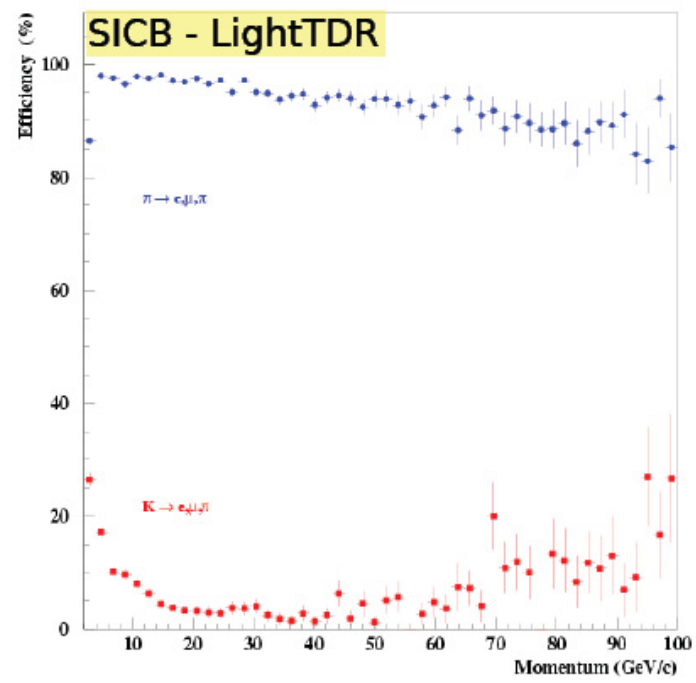

(3b)

Figure 3: The efficiency (in \%) of identifying pion as a light particle and the probability (in \%) of misidentifying Kaon as a light particle as a function of particle momentum for best measured track types. Figure (3a) uses data from GAUSS and Figure (3b) uses data from old simulation (SICB-LightTDR). The pion identification efficiency is above $75 \%$ in these figures. 
[2] W. Witzeling, Status of the LHCb Experiment. Proceedings of IEEE-NSS, Rome, 2004.

[3] S.Agostinelli et.al, 'GEANT4- a simulation toolkit', Nucl. Inst. Meth A 506(2003) 250303.

[4] M.Cattaneo et.al, Status of GAUDI eventprocessing framework, Proceedings of International Conference on Computing in High Energy Physics, Beijing, China, September 3-7, 2000.

[5] T.Sjostrand et. al, High-Energy Physics Event Generation with PYTHIA, Computer Physics Commun 135 (2001) 238.

[6] D.Lange et. al, The EvtGen Event Generator package, proceedings of International Conference on Computing in High Energy Physics, Chicago, USA, August 31September 4, 1998.

[7] G.Corti et. al, Software for the LHCb Experiment, Proceedings of IEEE-NSS, Rome, 2004.

[8] I.Belyaev et.al, Integration of GEANT4 with the GAUDI framework, Proceedings of International Conference on Computing in High Energy Physics, Beijing, China, September 3-7, 2000.

[9] T.Bellunato et. al, 'Performance of Aerogel as Cherenkov Radiator', Nucl. Inst. Meth. A 519 (2004) 493-507.

[10] R.Forty, RICH Pattern recognition for LHCb, Nucl. Inst. Meth. A 433 (1999) 257261. 